\title{
VAMOS NASSARAS: CAMBIO DE CÓDIGO COMO EJEMPLO DE LA APROPIACIÓN DE LENGUA EN CONVERSACIONES ESCRITAS
}

\author{
Carmen PÉREZ-SABATER \\ Universitat Politècnica de València (España)
}

\section{RESUMEN}

Este artículo trata de la apropiación del español por parte de comunidades en línea formadas por aficionados al fútbol de Camerún. El análisis de un corpus de comentarios publicados en redes sociales ha consistido en dos fases. Tras un primer estudio, cuyo objetivo ha sido identificar las lenguas más habituales de interacción en estas comunidades de aficionados africanos, se ha realizado un análisis discursivo de los mensajes con cambio de código al español que documenta sus funciones más frecuentes. Los resultados muestran que, en este espacio translingual, los comentarios escritos mayoritariamente en francés incluyen léxico en español para representar ciertas funciones discursivas como la alineación o el afecto; también se elige el español para configurar el nombre que algunos aficionados eligen en redes sociales. La apropiación discursiva del español fuera de su ámbito lingüístico habitual es un hecho constatado en el discurso deportivo recopilado para esta investigación, lo que es novedoso y de interés para la sociolingüística del español.

PALABRAS CLAVE: análisis del discurso electrónico; cambio de código al español; apropiación de lengua; multilingüismo en línea.

\section{AbSTRACT}

This article analyses the appropriation of Spanish by online communities of Cameroonian football supporters. The study of a corpus of online commentaries published on social networking has consisted of two distinct phases. After carrying out an analysis to identify the languages used in the commentaries, a detailed discourse analysis has been performed with special focus on the functions implied by those messages that included code-switching to Spanish. The results show that, in this translingual space, these comments include words in Spanish in a French-based text to represent certain discursive functions such as alignment or affect. The present research has aimed to shed light on the discursive appropriation of Spanish by African non-Spanish speaking communities, which has 
been corroborated in the sports discourse data compiled for the analysis, and is therefore newsworthy as well of interest for the study of Spanish sociolinguistics.

KEYWORDS: computer-mediated discourse analysis; code-switching to Spanish; linguistic appropriation; online multilingualism.

Fecha de recepción: 20/05/2020

Fecha de aceptación: 15/10/2020

Fecha de la versión definitiva: 22/10/2020

COMENTARIO 1. VAMOS LOS BLANCOS (Nassaras, término en fulfuldé o bassa, lenguas nativas de Camerún, que significa 'blancos', como se conoce a los jugadores de la Union Sportive de Douala por su equipación)

\section{9) Salomon VAMOS NASSARAS}

\section{INTRODUCCIÓN}

Dentro del análisis crítico del discurso, Fairclough $(2003,2013)$ habla de apropiación discursiva cuando un tipo de discurso ha colonizado otros tipos de discurso o bien cuando otros discursos se han apropiado de un tipo de discurso. En su caso, el tipo de discurso al que se refiere es el discurso económico de lo que Fairclough denomina el nuevo capitalismo o capitalismo contemporáneo. El proceso dialéctico de colonización y apropiación discursiva puede tener diferentes consecuencias. De estas, Fairclough (2013) destaca que la lengua local que se emplea para la gestión empresarial está colonizada por el discurso que denomina nuevo discurso global empresarial. Dejando a un lado las particularidades del discurso empresarial actual y adoptando un enfoque más general, Mar-Molinero (2006) indica que la mayoría de las publicaciones sobre la colonización y apropiación de lenguas han examinado, inevitablemente, el impacto de la lengua inglesa en otras lenguas, así como su expansión global y la repercusión que este proceso globalizador ha tenido sobre las lenguas minoritarias. En cuanto al español y su expansión globalizadora actual, este proceso de apropiación o expansión se ha estudiado primordialmente en los casos en que el español es una de las lenguas de interacción diaria, bien sea porque se analizan comunidades bilingües o porque se trata de zonas fronterizas donde está en contacto con otras lenguas (Mar-Molinero 2006). No se ha estudiado la repercusión del español en los intercambios comunicativos de comunidades o países donde esta lengua no se usa en una comunidad relativamente significativa, el tema principal de este artículo que se detalla a continuación. 
Por otra parte, Fairclough también comenta que este proceso de colonización y apropiación lingüística conlleva la mezcla de tipos de discurso y registros, hecho que se ha acelerado en los intercambios comunicativos realizados en la red (Baron 2011; Pérez-Sabater y Maguelouk-Moffo 2018) y que ahora parece incluir la mezcla de lenguas. Siguiendo estas líneas de investigación, este artículo analiza la apropiación del discurso futbolístico, principalmente de España, por parte de internautas de Camerún mediante el uso del cambio de código (CC), en un intento de alinearse con el discurso del éxito en esta disciplina deportiva. Como indica Androutsopoulos (2013), en la comunicación electrónica, la alineación es una de las funciones prominentes del cambio de código. Se entiende por CC, siguiendo a Gumperz (1977), la yuxtaposición dentro del mismo intercambio comunicativo de partes del discurso que pertenecen a dos sistemas o subsistemas gramaticales diferentes, aunque esta definición la matizaremos más adelante en la revisión y adaptación de la terminología sobre multilingüismo y discurso electrónico.

La investigación presentada aquí surge de un análisis anterior sobre el cambio de código y la mezcla de lenguas en intercambios publicados en redes sociales de Camerún, donde los resultados mostraron que las referencias al fútbol español son constantes (Pérez-Sabater y Maguelouk-Moffo 2019). Este artículo profundiza en lo esbozado anteriormente y se centra exclusivamente en la apropiación, por parte de aficionados cameruneses, del discurso futbolístico de sus equipos europeos preferidos; en concreto, de las lenguas oficiales de estos clubes cuyos partidos se siguen semanalmente a muchos kilómetros de distancia del país donde se juegan. A tal fin, se analizan comentarios publicados en las páginas de Facebook oficiales de los equipos de fútbol de Camerún más laureados para examinar qué funciones representan la inclusión de léxico en catalán, español e italiano ${ }^{1}$ en textos escritos primordialmente en francés por aficionados al fútbol de Camerún en sus publicaciones en línea. Hatalong (2015) señala que el español no es una lengua de comunicación habitual en el país, tampoco se emplean ni el catalán ni el italiano en ninguna de sus comunidades lingüísticas. La novedad del artículo radica básicamente en que identifica y clasifica el cambio de código al español fuera de su ámbito lingüístico habitual. Los ejemplos que se muestran dan fe de su influencia y de sus funciones más habituales en el discurso deportivo en redes sociales.

Antes de detallar el marco teórico, el corpus y la metodología del análisis, es necesario explicar brevemente el contexto lingüístico y cultural de Camerún para comprender mejor el trabajo que se expone.

1 Aquí, por orden alfabético, más tarde, por prelación. 


\section{Contexto del estudio}

Camerún, país de África occidental, ha sido colonizado por varias potencias europeas a lo largo de su historia. A consecuencia de sus sucesivas colonizaciones y reagrupaciones territoriales, el país está dividido en dos zonas lingüísticas diferenciadas desde que se independizó de Francia en los años 1960: la zona anglófona, donde el inglés es la lengua oficial, y la zona francófona, mayoritaria en el país, donde es el francés. Sin embargo, a pesar de la doble oficialidad, el francés prevalece sobre el inglés en la vida pública de Camerún (Atanga 2011). La rivalidad lingüística provoca enfrentamientos políticos entre las dos comunidades lingüísticas que llegan a convertirse en oleadas de revueltas armadas cada pocos años (Anchimbe 2013). Junto con las dos lenguas oficiales, en Camerún se hablan más de 240 lenguas indígenas de familias lingüísticas africanas diferentes como bamileké, bantú o beti (Mulo 2011). A estas hay que añadir varias lenguas criollas surgidas en los últimos años en las zonas urbanas del país, como sucede en muchas ciudades de África (Simpson 2008). Las lenguas criollas más populares son el pidgin inglés, mezcla de inglés informal, lenguas indígenas y francés, y el camfranglais, que sigue la sintaxis del francés, pero incorpora léxico del inglés y lenguas autóctonas. Como señalan Ayafor y Green (2017), las criollas sirven de lenguas francas en gran parte del territorio camerunés junto con algunas de las lenguas indígenas como el fulfuldé. La mayoría de las lenguas autóctonas y criollas no están estandarizadas y no tienen tradición escrita, solo las dos lenguas oficiales se enseñan, se escriben y se emplean en la administración y entornos formales (Mulo 2011). Como resultado de este complejo panorama lingüístico, un camerunés se comunica diariamente en varias lenguas según su intención comunicativa y el medio de comunicación: usará una de las dos lenguas oficiales en la administración y en los centros educativos, una o varias lenguas indígenas cuando interactúe con su familia y una de las lenguas criollas populares para comunicarse en la calle y los mercados (Atanga 2011). Como rasgos característicos de la sociedad camerunesa cabe señalar que es multilingüe, está dividida en dos grandes comunidades lingüísticas, la anglófona y la francófona, y, sobre todo, que es una sociedad donde la mezcla de lenguas y la creatividad léxica están a la orden del día en la conversación (Mulo 2011).

Camerún presenta muchas deficiencias en cuanto al desarrollo empresarial y tecnológico. Al respecto, hay que decir que Internet solo llega al $24 \%$ de sus habitantes (<https://www.internetworldstats.com/stats $1 . h t m>$, 02/02/2020). Como apunta Kytölä (2013), la baja penetración de Internet en la sociedad repercute claramente en un menor acceso a la educación. Los datos estadísticos y los estudios sociólogos sobre este país africano nos muestran que los cameruneses son unos apasionados de Facebook -el $50 \%$ 
de la población con acceso a Internet tiene una cuenta en esta red socialy del fútbol, considerado el deporte rey de Camerún y de toda África (Vidacs 1999). Por tanto, el estudio de interacciones en Facebook sobre fútbol nos puede dar una idea clara y real de cómo se comunican sus habitantes en las redes sociales.

Actualmente, el fútbol es, para gran parte de la población, el deporte global, como señala Antezana (2003). Este deporte nació en Inglaterra, sus reglas se definieron allí y desde Gran Bretaña se expandieron al resto del mundo. Las particularidades del lenguaje futbolístico en español se han estudiado desde diversas perspectivas y metodologías. De especial relevancia son los estudios terminológicos que analizan los neologismos en el fútbol procedentes del inglés y francés (ver, por ejemplo, Aleixandre-Benavent et al. 2007) y su influencia en el discurso periodístico. Como indica Hernández (2012), en España, la inclusión de palabras extranjeras en los titulares hace que estos sean más atractivos, exóticos y llamativos para el lector. Recientemente, las publicaciones sobre el discurso del fútbol han tomado caminos diferentes en línea con los cambios experimentados por el llamado deporte rey y su expansión global como empresa de entretenimiento (Ginesta et al. 2020). En relación con esto, Hernández (2012) argumenta en su libro sobre el lenguaje deportivo actual que el aficionado especialista de antaño ha pasado a ser el aficionado global, a quien, en muchos casos, solo le interesa la espectacularidad del deporte como acontecimiento de moda. Las victorias de la selección española en la Eurocopa (2008, 2012) y el Mundial (2010), pero, principalmente, las victorias de los equipos españoles en las ligas europeas junto con las políticas de expansión de estos clubes hacia el exterior han puesto de moda a las escuadras españolas. Esto es así, sobre todo, en cuanto al Barcelona FC y al Real Madrid CF (Crolley 2008; Onwumechili y Akindes 2014), que ocupan los primeros puestos por número de seguidores en las redes sociales (Jackson 2014).

No hay duda de que actualmente el fútbol conecta a personas de diferentes países y orígenes socioculturales (González Ramallal 2014). La repercusión del fútbol europeo en África y concretamente en Camerún es apabullante. La retransmisión de los partidos de la Premier League y LaLiga en directo vía satélite es vista por muchos como una segunda o nueva colonización del continente africano por parte de las potencias europeas, denominada colonización electrónica (Pannenborg 2012). Otro hecho importante que ha provocado el interés por las ligas europeas es que jugadores de élite del país o de ascendencia camerunesa como Mbappé han logrado ser figuras en clubes de Europa. Prueba de ello son las referencias constantes a clubes como el Arsenal, Barcelona FC, Manchester United o Real Madrid CF en blogs y foros sobre fútbol de Camerún. Por ejemplo, Pannenborg (2012) descubre en su análisis del mercado futbolístico africano que en el 
foro camerunés que analiza hay tantas referencias al Barcelona como al equipo nacional de Camerún. Sin embargo, el objetivo de este artículo no es tratar la repercusión del fútbol europeo en la economía africana como en Pannenborg (2012), sino la influencia del fútbol europeo en el país africano desde la lingüística. El enfoque lingüístico de este artículo aporta un punto de vista novedoso y puede subsanar el vacío existente en este campo de investigación. El discurso analizado en este estudio podría considerarse un colonizing discourse type, que, en parte, se evidencia mediante el cambio de código al español, italiano y catalán que se expone en este artículo. Podemos afirmar que el fútbol es un 'tipo de discurso colonizador' porque es un pilar importante en la vida diaria de mucha gente (Hernández 2012; Chiweshe 2014; Ginesta et al. 2020), cumpliendo, por tanto, con uno de los requisitos principales exigidos por Fairclough (2003) para identificar este tipo de discurso.

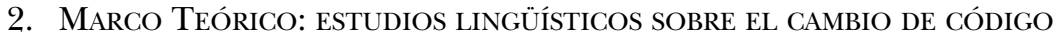 Y ELECCIÓN DE LENGUA EN INTERNET}

El multilingüismo, el cambio de código y la elección de lengua en Internet se comenzaron a estudiar en la década de 1990 (Georgakopoulou 1997). Sin embargo, no podemos decir que constituye una rama diferenciada dentro de la comunicación electrónica, en inglés, Computer-mediated Communication $^{2}$ (CMC), hasta bien entrada la década inicial del siglo XXI. En las primeras investigaciones, el multilingüismo y la elección de lengua adoptaron una perspectiva macrosociológica para averiguar las lenguas más usadas en la red (ver, entre otros, Warschauer y De Florio-Hansen 2003; Paolillo 2007). Más adelante, se adoptaron otras líneas de investigación basadas en la pragmática, el análisis del discurso y la sociolingüística con el fin de analizar e identificar el multilingüismo, adaptando los estudios clásicos sobre la mezcla de lenguas y el cambio de código a las nuevas prácticas discursivas en Internet (Leppänen y Peuronen 2012; Androutsopoulos 2015; Lee 2016). Se destacan aquí los aportes teóricos y metodológicos de las publicaciones sobre la elección de lengua y cambio de código más representativas hasta la fecha.

La revisión de términos y metodologías comienza con delimitar qué se entiende por multilingüismo en línea.

La definición de cambio de código acuñada por Gumperz (1977) debe ajustarse al análisis discursivo de las 'conversaciones escritas', written speech,

${ }^{2}$ En algún caso se citan términos en inglés por su interés académico, como en este caso, ya que CMC surgió en inglés y sobre esta lengua en la década de los años noventa. 
término que acuñó Maynor (1994) para nombrar los textos intercambiados gracias a la red. Androutsopoulos (2015) propone que el estudio del multilingüismo en la red se ciña a los intercambios entre personas o grupos que están conectados de forma digital mediante una conexión informática. Al respecto, Androutsopoulos (2013: 673) puntualiza que para hablar de cambio de código en comunicación electrónica debe haber pruebas de que «... [different language choices] are in some way dialogically interrelated by responding to previous, and contextualizing subsequent, contributions». Es decir, solo cuando varias lenguas se relacionan dialógicamente tendríamos cambio de código en comunicaciones en línea. Por tanto, se excluirían de la rama de la lingüística que estudia el discurso electrónico otras tareas como, por ejemplo, comprobar y medir las lenguas de una determinada página web plurilingüe. Así pues, no hablamos de multilingüismo cuando tenemos varias lenguas en la misma página web, ni tampoco hablaríamos de cambio de código aunque coexistan varias lenguas o variedades de una lengua en la misma plataforma de comunicación (Lee 2016).

En segundo lugar, se deben reconsiderar algunas de las propuestas terminológicas tradicionales sobre la mezcla de lenguas en la conversación que ahora no tendrían validez, como indica Lee (2017). Según esta autora, cuando hablamos de la elección de lengua en entornos digitales, no hablamos únicamente de las lenguas que un hablante conoce -como en el análisis de la conversación entre hablantes bilingües-, sino que su estudio debe tener en cuenta los códigos y recursos lingüísticos de los que disponen los participantes en línea, sin que sea relevante, en muchos casos, el nivel de conocimiento lingüístico de la lengua o lenguas que emplea el autor del mensaje o el conocimiento que los demás internautas tengan de esas lenguas. También hay que tener en cuenta que los participantes en una misma red social no siempre comparten estos códigos, lo que no suele impedir una comunicación fluida gracias a que, en muchas de estas plataformas de comunicación, se proporcionan herramientas que facilitan la traducción de cualquier texto a muchas de las lenguas que se hablan en el planeta. Muestra de ello es el hecho de que Facebook permite la traducción de cerca de 150 lenguas actualmente (<https://www.facebook.com $>$ ).

Además, un nuevo entorno de comunicación como es Internet exige nuevos términos y conceptos para explicar los fenómenos lingüísticos que tienen lugar en estos intercambios comunicativos. Principalmente, hay que hablar de las redes sociales como un 'espacio translingual', término aceptado en la investigación sobre mezcla de lenguas en conversaciones escritas (Pérez-Sabater y Maguelouk-Moffo 2019). Translanguaging es un concepto que Li (2011) adaptó de la educación bilingüe (García 2009) y que implica que un texto intercambiado en la red no llevaría la etiqueta de escrito en una lengua concreta, más bien tendríamos espacios virtuales 
(Chiweshe 2014) o 'translinguales', donde el uso de cualquier código, lengua o variedad de lengua depende únicamente de la intención comunicativa de cada autor (Pérez-Sabater y Maguelouk-Moffo 2019). Por el contrario, otras acepciones que se han acuñado en los últimos diez años no han conseguido captar la atención de los lingüistas en igual medida. Como ejemplo de propuestas terminológicas que, aunque han sido correctamente formuladas no han tenido repercusión académica, cabe mencionar el término acuñado por Hinrichs (2016) para referirse conjuntamente a la elección de lengua y cambio de código en la red, 'contraste de lenguas digital' o digital language contrasting en el original.

En lo que respecta a las funciones pragmáticas, discursivas y sociales de los llamados fenómenos lingüísticos de mezcla de lenguas, estas funciones se han tenido que ajustar a los intercambios en línea. A las funciones destacadas por los estudios clásicos sobre las conversaciones de hablantes bilingües (Gumperz 1977) -señalar citas, interjecciones, repetición, marcar a un interlocutor determinado o indicar la intención de pertenencia o exclusión a un grupo concreto (we code en oposición a you code), etc.-, en los intercambios mediante la red añadimos el cambio para realizar fórmulas discursivas o para enfatizar o remarcar prácticas culturales específicas (por ejemplo, ver Montes-Alcalá 2016). Androutsopoulos (2013) recoge las funciones discursivas del cambio de código de publicaciones anteriores y elabora una lista con ocho funciones discursivas principales; de estas se destacan aquí las relacionadas con indicar acuerdo o desacuerdo, consentimiento o disentimiento, alineación o distancia. Como explica Androutsopoulos (2013), un análisis discursivo de textos con cambio de código puede mostrar cómo el uso de varias lenguas en un mismo intercambio comunicativo representa una de estas funciones y valores de identidad para una determinada comunidad o grupo de internautas.

Para concluir este apartado, se tratan los estudios lingüísticos que han abordado el cambio de código y la elección de lengua en intercambios en español o donde el español es una lengua propia de la comunidad estudiada, donde normalmente la lengua minoritaria sirve para marcar la identidad grupal frente a la lengua mayoritaria. Estos se limitan a los artículos de Negrón-Goldbarg (2009) y Montes-Alcalá (2016) sobre la mezcla de español e inglés en correos electrónicos de comunidades bilingües en EE.UU. y a las tesis y artículos derivados de estas de Alba (2015) y Arias (2017) sobre el contacto lingüístico del español y el asturiano en la red. Estas publicaciones son pocas teniendo en cuenta que el español es una de las lenguas con más hablantes en el mundo, si las comparamos con el interés suscitado por el tema por parte de lingüistas de otras lenguas con menor alcance y hablantes, por ejemplo, el griego (ver Tsiplakou 2009). La explicación de esta ausencia de publicaciones excede el objeto de este trabajo, dado que 
aquí no examinamos los intercambios escritos en grupos o comunidades bilingües donde el español, italiano o catalán son lenguas diarias de interacción, sino de intercambios en los que los internautas no son hablantes habituales de estas lenguas. Hasta el momento, no existen publicaciones sobre el cambio de código al español en comunidades de países donde esta lengua no es de uso diario, de ahí, la trascendencia de la investigación realizada para este artículo.

\section{Corpus y METOdología}

\subsection{Corpus}

El corpus se ha formado a partir de todos los comentarios publicados en Facebook durante 2019 en las páginas oficiales de los equipos que han ganado la liga de fútbol de Camerún en los últimos diez años. El palmarés de la denominada Elite One desde 2010 está encabezado por el Coton Sport de Garoua (CSG) (6 ligas), Union des Mouvements Sportifs de Loum (UMS) (2 ligas), Union Sportive de Douala (USD) y Ending Sport Football Club de la Lékié (1 liga cada uno). Debido al escaso desarrollo tecnológico del país, el conjunto que más títulos ha conseguido desde 2010, Coton Sport, tiene solo unos 12000 seguidores en esta red social ${ }^{3}$. Los comentarios de estos clubes no eran suficientes para este estudio y se recurrió a la página Lion Indomptable, de donde se recopilaron más ejemplos publicados en otoño de 2019. Esta página de Facebook de libre acceso con 80000 seguidores informa de las noticias de la selección nacional camerunesa y de sus jugadores más carismáticos; su nombre hace referencia a 'Los Leones Indomables', Lions Indomptables, como se conoce a la selección de Camerún (Vidacs 1999). De estas fuentes, se ha seleccionado un total de 457 comentarios públicos (2283 palabras), tras excluir los mensajes formados exclusivamente por resultados de partidos, gifs o emoticonos. Los textos son públicos, es decir, no hay que subscribirse a las páginas para consultarlos, pero, aun así, se muestran anonimizados. Además de comentarios, se han estudiado los nombres que los autores de los comentarios han elegido en Facebook para identificarse porque muchos contienen palabras en español o referencias al fútbol español. En estos casos, los ejemplos conservan el nombre completo del seguidor camerunés tras haber obtenido permiso por escrito de estos participantes mediante la aplicación Messenger de Facebook.

\footnotetext{
${ }^{3}$ Los dos equipos españoles más laureados, Barcelona FC y Real Madrid CF, reúnen a 103 y 104 millones de seguidores respectivamente en sus páginas oficiales de Facebook en diciembre de 2019.
} 
Mostramos un comentario de la página Lion Indomptable donde se aprecia el valor que los hinchas del país africano dan a los equipos españoles, en este caso, al Barcelona FC. Este texto se publica en la página dedicada a animar a los Leones Indomables antes de un partido internacional. Uno de los aficionados se queja de que ninguno de los integrantes de la selección camerunesa sirve para jugar al fútbol porque ninguno juega en el Barça.

Comentario $2^{4}$. Ninguno de ellos (se refiere a los jugadores de la selección actual de fútbol de Camerún) juega en el Barça, por tanto, no valen (Lion Indomptable)

Oumarou Aucun d'entre eux ne joue pour le Barça dont ils
sont nuls
...

Muchos fans africanos se identifican con equipos de sus países, pero, a la vez, con europeos, a los que consideran más profesionales y de juego más ameno, según constata Pannenborg (2012). Como muestra de la afición por los clubes europeos, hay un hecho que se suele citar en las publicaciones sobre fútbol en el continente africano: en 2009, en la celebración de la victoria del Barça contra el Manchester United, un fan del Manchester atropelló con un autobús a un grupo de culés que estaban celebrando la victoria de su equipo. Murieron 4 fans. Tanto las víctimas como el conductor eran de Nigeria y vivían en ese país (Pannenborg 2012).

\subsection{Metodología}

El análisis ha consistido en dos fases bien diferenciadas.

En primer lugar, siguiendo a Tsiplakou (2009), se contaron las palabras en cada lengua del total de vocablos en todos los comentarios, 2283 palabras. Es necesario aclarar que en este proceso no se contabilizaron los emoticonos ni los emojis porque normalmente se cuentan como palabras, a propuesta de Baron (2008), pero aquí es imposible que se asignen a una lengua determinada. Los resultados del primer análisis se muestran en porcentajes en la Tabla 1, ya que las cifras pueden reflejar mejor el alcance real del uso de las lenguas en cuestión, catalán, español e italiano, en el total del corpus, si se presentan en comparación con las lenguas oficiales y autóctonas de Camerún. mejor.

${ }^{4}$ En ocasiones se añade puntuación a la traducción para que el texto se comprenda 
Leppänen y Peuronen (2012) señalan que los estudios cuantitativos sobre el multilingüismo en línea se deben completar con otros bajo perspectivas y metodologías distintas. Por consiguiente, en una segunda fase, se seleccionaron los comentarios donde había léxico en catalán, español e italiano para examinar las funciones discursivas que el uso de estas lenguas extranjeras conlleva en cada caso concreto. Como propone Herring (2007) en su método de análisis del discurso electrónico (Computer-mediated Discourse Analysis, $C M D A$ ), la elección de una lengua determinada en el Internet multilingüe tiene una función discursiva específica. Para seleccionar las funciones sobre la heterogeneidad lingüística en Internet se siguió a Tsiplakou (2009), Androutsopoulos (2013), Montes-Alcalá (2016) y Georgalou (2017). De todas sus propuestas, el análisis se centra en el CC para las expresiones de afecto, énfasis y para crear un determinado efecto pragmático. Además, se recurrió a Hernández (2003 y 2012) y Guerrero (2018) para el análisis del léxico futbolístico y sus metáforas.

Por último, ante el elevado número de nombres de Facebook que incluyen palabras en español o referencias a equipos o jugadores españoles, se decidió llevar a cabo un análisis de los nombres de los aficionados en esta red social, cuyos resultados también se expondrán con varios casos al final de la sección.

\section{RESUlTAdOS Y SU INTERPRETACIÓN}

Los resultados del primer análisis que se exponen en la Tabla 1 confirman que las intervenciones analizadas están mayoritariamente en francés, la lengua base o matriz más frecuente en el corpus. Esto es así no solo porque el francés es la lengua oficial principal del país sino también porque los equipos que han ganado la Elite One en los últimos diez años pertenecen a las regiones francófonas.

La mayoría de los comentarios son multilingües -el $72 \%$ incluyen palabras en varias lenguas o variedades de una lengua-, siendo el inglés la segunda lengua más frecuente, generalmente para fórmulas discursivas. El corpus incluye, además, léxico en lenguas indígenas y en otras lenguas europeas diferentes del francés o inglés, principalmente en español, pero también en italiano y catalán. De este último grupo solo hay una oración completa y está en español. Antes de pasar al segundo análisis, hay que explicar que los resultados obtenidos son del corpus recopilado en el año 2019, un corpus compilado en otro momento podría arrojar resultados diferentes.

El $3 \%$ del total de las palabras del corpus está en español, italiano y catalán. Las 69 palabras en estas lenguas podrían parecer pocas, pero este dato de ninguna manera puede pasar desapercibido si tenemos en cuenta que se han analizado todos los comentarios publicados en las páginas de 
TABLA 1. Porcentaje de palabras en cada lengua en el corpus

\begin{tabular}{lcc}
\hline \multicolumn{1}{c}{ Lengua } & Número palabras & Porcentaje \\
\hline Francés & 1645 & $72 \%$ \\
\hline Inglés & 410 & $18 \%$ \\
\hline Lenguas indígenas camerunesas & 159 & $7 \%$ \\
\hline $\begin{array}{l}\text { Español, italiano y catalán } \\
\text { (por orden de prelación) }\end{array}$ & 69 & $3 \%$ \\
\hline
\end{tabular}

los mejores clubes del país y que estamos hablando de lenguas que no son propias de ninguna comunidad lingüística de Camerún.

Estos casos se clasifican en 3 grupos: CC al español, italiano y catalán para animar al equipo, CC para términos del lenguaje futbolístico y CC para expresiones de afecto. El último punto de este apartado trata los nombres de los participantes en Facebook.

\subsection{Cambio de código al español, italiano y catalán para animar al equipo}

En este apartado se muestran los casos más habituales de CC en los comentarios de la Elite One y los Leones Indomables: la inclusión de los lemas o expresiones nacidos en las gradas, en las peñas o presentes en el himno del club que acompañan a un equipo, selección o jugador de España o Italia. Las expresiones vamos, adelante, viva, forza, força o hala se emplean con regularidad en comentarios mayoritariamente en francés.

En primer lugar, se ilustran los resultados con los ejemplos de CC al catalán con la expresión de ánimo del Barça:

Comentario 3. Ánimo, campeón (Ending Sport)

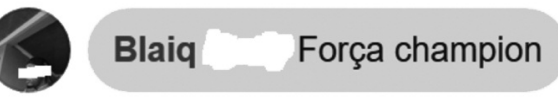

Blaiq anima al equipo ganador de la liga africana usando la expresión que suele acompañar al Barcelona, palabra en catalán que se incorporó al club durante la transición española (Llopis-Goig 2008).

Otros casos donde se ha utilizado força o viva no son ejemplos de CC porque el comentario está formado exclusivamente por una sola palabra en catalán, español o italiano y el nombre del equipo; no hay, por tanto, alternancia de lenguas. 
Comentarios 4, 5, 6. CSG

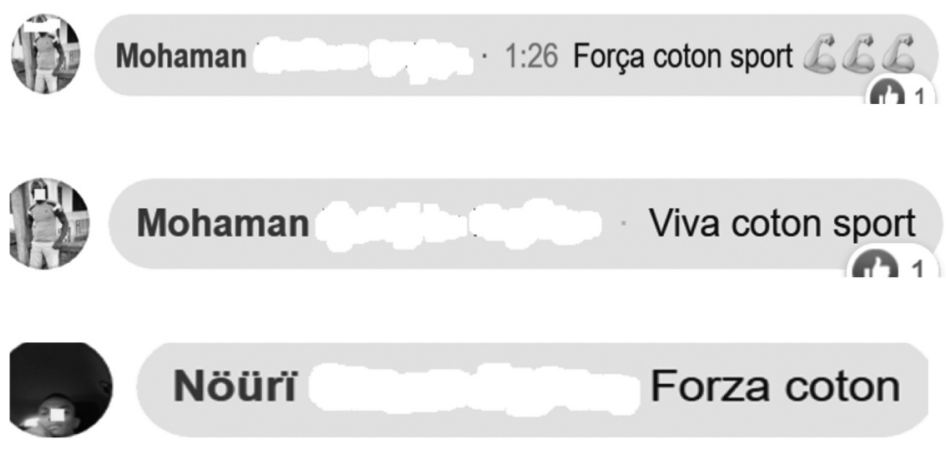

El caso de Mohaman (Comentarios 4 y 5) es representativo de las prácticas discursivas camerunesas en redes sociales sobre fútbol: la afición usa indistintamente estas expresiones del Barcelona, de la selección española o de la italiana para animar al mismo equipo camerunés, Coton Sport de Garoua, ante un encuentro próximo. Los cameruneses copian lo que escuchan en las retransmisiones vía satélite o leen en las redes sociales, ya que seguramente muchos de estos aficionados siguen en paralelo a clubes europeos (Pannenborg 2012). Probablemente, los autores desconocen qué lengua están empleando o la ortografía correcta de estas expresiones. Esto es así, sobre todo, en los casos de força o forza porque tanto su pronunciación como su ortografía son parecidas en catalán e italiano. Seguidores de la página de Facebook del Barcelona dudan frecuentemente de la escritura de força (Pérez-Sabater y Maguelouk-Moffo 2019).

En comentarios formados por una oración completa, se aprecia CC a estas lenguas románicas mediante expresiones como vamos, hala o siempre adelante, normalmente insertadas en comentarios en francés.

COMENTARIO 7. Vamos Maestro Tchuente y Namekong Rostan, sois unos cracks (CSG)

\section{Landry Vamos Maestro Tchuente et Namekong Rostan vous etes des cracks}

Landry usa el español para subir la moral del equipo ante una confrontación deportiva, vamos, dirigida al entrenador y un jugador del equipo camerunés más laureado. Este aficionado considera el español como lengua de éxito en este deporte y así lo demuestra en su texto. 
En el Comentario 8, destaca el CC al español como fórmula discursiva para despedir el texto:

COMENTARIO 8. Mi bombón, equipo fuerte, sois una referencia.

Hala Coton. Siempre adelante (CSG)

Armelle Charless
êtes la reference. Hala Coton $:$ Siempre adelante

Armelle comienza su intervención de ánimo en francés coloquial o camfranglais, sigue en inglés, francés y termina exhortando al equipo de Garua en español con el lema del Real Madrid, que proviene de su himno (Hernández 2012). La autora despide el texto en un español correcto, que puede haber aprendido en el colegio, dado que gran parte de los internautas cameruneses son jóvenes con educación (Penard et al. 2015). Su nivel medio de instrucción viene reflejado por la correcta organización textual y el dominio de las destrezas escritas en varias lenguas. El comentario está bien separado por oraciones, aunque no siempre mediante la puntuación adecuada. Tanto la frase nominal en español como la adverbial final incluyen mayúsculas y puntuación correcta.

En la misma línea, el Comentario 9 demuestra el dominio del español a nivel escrito:

Comentario 9. Adelante, UMS de Loum. Estoy muy orgulloso de mi equipo. Esta es vuestra temporada. La victoria guiará vuestro camino siempre. Queremos que el equipo nos dé otra copa. Adelante. Mis mejores deseos ${ }^{5}$

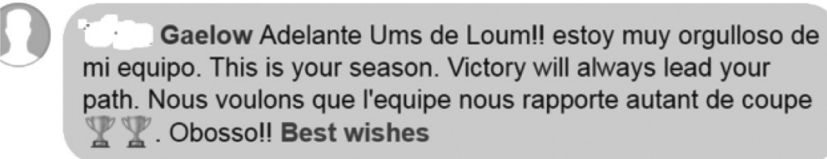

El aficionado del Ums emprende su intervención multilingüe en español con el adverbio Adelante, clara metáfora de guerra en el fútbol (Hernández 2012), seguido por la única oración completa en español del corpus que tiene como objetivo expresar la admiración del autor por su equipo local. El texto sigue en inglés y francés, repitiendo la expresión de aliento inicial en una lengua autóctona, lengua duala, con obosso ('adelante'). El doble

\footnotetext{
${ }^{5}$ Agradezco a la Dra. Ginette Maguelouk-Moffo sus traducciones al español de los términos en lenguas indígenas camerunesas.
} 
CC al español y duala con el mismo significado y en dos lenguas distintas de las dos lenguas oficiales que conforman mayoritariamente este comentario, inglés y francés, enfatiza doblemente el deseo de victoria, una de las funciones más relevantes del CC en redes sociales según Androutsopoulos (2013) y Georgalou (2017). Este comentario es un claro ejemplo del multilingüismo también imperante en las prácticas escritas actuales por Internet de Camerún (Pérez-Sabater y Maguelouk-Moffo 2019).

\subsection{Cambio de código para términos del lenguaje futbolístico}

El léxico especializado asociado con el deporte se ha considerado siempre uno de los rasgos principales del discurso deportivo junto con una prosodia especial y unas características sintácticas diferenciadas (Ferguson 1983; Pérez-Sabater et al. 2008; Hernández 2012). Esta investigación se centra en el CC al léxico especializado del deporte en catalán, español e italiano. Los usos trasladados son las palabras más habituales en el lenguaje deportivo, es decir, léxico de otros campos empleado en el deporte para dar brillantez al discurso, transmitir expresividad y seducir y cautivar al oyente o lector; entre estos, hay que destacar las metáforas (Hernández 2003; Guerrero 2018). En el corpus son frecuentes las metáforas lexicalizadas, mercato en italiano para referirse al traspaso o 'mercado' de jugadores, pero, sobre todo, el epónimo pichichi, habitualmente con variada ortografía. Normalmente, este término se emplea para elogiar a un jugador, pero, en algún caso, también a una jugadora de la selección femenina. Como indican Aleixandre-Benavent et al. (2007), pichichi está generalizado ya en español incluso fuera del léxico futbolístico y se recoge en la Real Academia. Como novedad, se aprecia aquí que su uso está generalizado también en Camerún.

El Comentario 10 comienza a ilustrar esta categoría:

Comentario 10. El Coton Sport debe encontrar en el mercado 2 defensas para evitar la porosidad después del final del campeonato (CSG)

Roger - Coton Sport doit trouver pendant le mercato 02 défenseurs pour stopper la porosité de la défense depuis début du championat

Mercato es usual en el corpus para referirse al mercado futbolístico, término arraigado ya en francés para denominar el trasvase de jugadores de un equipo a otro y que forma parte del título de varias páginas de Internet sobre el tema con muchos seguidores. 
Otro término común en los comentarios es la palabra italiana squadra para referirse al equipo, en el Comentario 11 con error. En este caso, la lengua base es el inglés, la otra lengua oficial de Camerún.

COMENTARIO 11. Tiene que estar en el equipo senior (se refiere a un jugador de la selección sub21) (Lion Indomptable)

Las metáforas usuales o lexicalizadas propias del deporte sirven normalmente para captar la atención del lector o del oyente (Hernández 2003) a modo de cursiva o subrayado. El caso de remontada en el Comentario 12 cumple esta función y añade dramatismo al juego. Se inserta en un texto en francés con un CC que parece remarcar aún más el esfuerzo que debe hacer el equipo.

Comentario 12. Sí, sí, imposible no es el 'lumonés'... ¿es esta la remontada que queréis ver? (UMS)

\section{Edmond Ouais ouais impossible n'est pas loumois...c'est
la remontada que vous voulez voir?}

Por último, el Comentario 13 puede ilustrar tanto esta categoría como la siguiente y puede servir de enlace entre ambas. Aquí, pichichi se emplea para alabar a una jugadora, es un CC en un texto mayoritariamente en francés con una oración final en pidgin inglés. La pichichi es, en esta intervención, la máxima goleadora de la selección femenina de fútbol de Camerún.

COMENTARIO 13. Bravo la pichichi Ajara Nchout, la campeona del equipo femenino camerunés.

Eres la mejor, hermana mayor (Lion Indomptable)

\section{* Toton Pong teh la pichichi Ajara Nchout, la championne de} l'équipe feminine camerounaise. You be na de best big sister

Encabeza el comentario una expresión en gombaleu, lengua del oeste camerunés, que significa 'bravo'. Toton sigue elogiando a la delantera más internacional de la selección femenina, Ajara Nchout, con CC al español 
mediante pichichi; en 2020 esta futbolista juega en la liga femenina noruega. No hay duda de que la persona que escribe el texto sabe español, al menos a nivel básico, porque distingue entre el artículo masculino y femenino, posiblemente ha aprendido español en el colegio como seguramente los autores de los Comentarios 8 y 9 . El texto termina en pidgin inglés, una de las lenguas criollas más extendidas en el territorio camerunés, con be y $n a$, dos palabras que se refieren al verbo ser, $b e$, en segunda persona, 'eres'. En el análisis del pidgin inglés de Camerún, Ayafor y Green (2017) destacan que la repetición de palabras con significante diferente, pero con el mismo significado, es una de las características más sobresalientes de esta lengua criolla. El comentario termina con una fórmula de cortesía marcadamente camerunesa que incluye léxico relacionado con la familia en señal de respeto y para establecer solidaridad grupal y étnica, según indica Anchimbe (2013).

\subsection{Cambio de código para expresiones de afecto}

El lenguaje del deporte tiene tendencia a la exageración, al dramatismo, la emotividad, la alabanza desmedida (Hernández 2003). En los casos que se muestran a continuación, esta alabanza desmedida o dramatismo se realiza frecuentemente en una lengua distinta de la lengua base o matriz (Pérez-Sabater y Maguelouk-Moffo 2020), en este corpus, se elige el español en muchos casos.

En esta categoría hay dos grandes grupos de ejemplos. En primer lugar, se analizan los que incluyen elogios hacia un jugador/a ejemplar con términos relacionados con la familia:

COMENTARIO 14. Bienvenido hermano, me gusta la alternancia que favorece la diversidad. Es una temporada de victoria. El fútbol es el fútbol (USD)

Irene Welcome hermano j'aime cette alternance dans l'équipe qui favorise la diversite C'est une saison de victoire Football na damba $\quad: \because: \lambda^{3}$ 国

Este texto multilingüe combina varias lenguas: primero inglés, seguido de español para dirigirse afectuosamente al jugador, francés y fórmula de despedida en inglés y pidgin inglés, Football na damba, 'el fútbol es el fútbol', combinación léxica popular en el país (Pérez-Sabater y Maguelouk-Moffo 2019) y que, de nuevo, utiliza la repetición de léxico, en este caso, sustantivos. 
Un segundo grupo engloba casos donde jugadores o entrenadores son los maestros, término coincidente en español e italiano.

COMENTARIO 15. Bravo mi hermano Tchuente Thierry (maestro 13), hace falta que mi otro hermano Yimga Didier marque (CSG)

Fc le Dou Bravooo mon brother Tchuente Thierry (maestro 13) il faut aussi que mon autre brother Yimga Didier marque

En esta intervención, es de destacar el CC al inglés para los elogios mediante términos familiares como en Anchimbe (2013). El español/italiano son los códigos del éxito y se emplean para un cumplido mayor: el maestro, el que más sabe.

\subsection{Nombres en Facebook}

En la actualidad, es patente el hecho de que el periodismo deportivo ha contribuido a que los logros de los atletas se proyecten hacia la comunidad a la que representan, haciéndola partícipe de un éxito colectivo (Rojas 2012). Los jugadores de fútbol son considerados héroes y divinidades a los que seguir, son «el mejor exponente de la realización personal y social» y sus nombres son esenciales en el lenguaje deportivo porque son los que sirven para identificar a los héroes o los culpables de la derrota, de ellos depende el éxito o el fracaso en el campo (Hernández 2012: 25).

En el último grupo de ejemplos, la identificación con equipos españoles es de especial interés. Los internautas eligen nombres de jugadores, palabras en español o italiano y nombres identificadores de estos clubes para conformar su nombre en la red social Facebook. Al igual que otras redes como Instagram o TikTok, los nombres en Facebook permiten revelar información personal real o crear y mostrar otra identidad inventada (Papacharissi 2009). La identificación con lengua y éxito en esta disciplina deportiva es aquí aún más significativa: los aficionados quieren conseguir ser tan excelentes como su equipo o jugador preferido y para ello se apropian de su nombre o referente.

Cabe destacar en el corpus varios tipos de agrupaciones léxicas.

El Recorte 1 representa al grupo de internautas que adoptan el nombre completo de un jugador español o que ha jugado en LaLiga para formar su propio nombre en Facebook. Con cierta frecuencia, se completa con léxico en español siempre de carácter positivo, lo que refuerza la admiración que el aficionado tiene por su ídolo. En estos casos, no habría CC ni mezcla de lenguas. 
RECORTE 1. Seguidor de la página Lion Indomptable

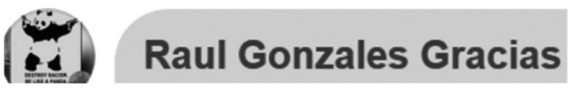

Este admirador de Raúl González, futbolista del Real Madrid entre 1994 y 2010, añade el indicador de cortesía, gracias, al nombre. En cierta forma, su nombre completo en Facebook es un indicador de cortesía en sí mismo hacia Raúl.

En otras ocasiones, como indica el Recorte 2, se observa otra combinación léxica: el apodo con el que se conoce al jugador, normalmente en el caso de futbolistas latinoamericanos (Hernández 2012). Aquí, el internauta probablemente añade su nombre real (eliminado para mantener la privacidad) al del internacional mexicano Javier Chicharito Hernández, otrora jugador del Madrid, junto con el adjetivo sustantivado de carácter laudativo El Dorado (Briz 1990).

RECORTE 2. Seguidor Lion Indomptable

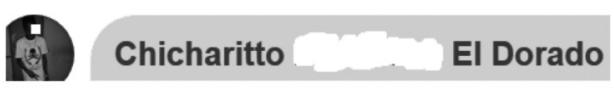

La agrupación léxica del Recorte 3 está formada por el apellido de un futbolista español, Fernando Torres, junto con el nombre del internauta y un término léxico positivo, pero esta vez en inglés, Power:

RECORTE 3. Seguidor del UMS

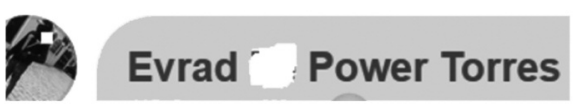

En los Recortes 4 y 5, no figuran nombres propios de deportistas, sino sustantivos positivos del lenguaje futbolístico en español o italiano que los aficionados adoptan para su identificación en esta red social.

ReCorte 4. Seguidor del CSG

\section{Moustafa Maestro Mai}

O pichichi en ortografía no estándar. 
ReCORTE 5. Seguidor del CSG

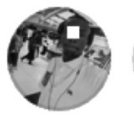

\section{BI le Pitchitchi}

Finalmente, aunque aquí no siempre se puede hablar de fenómenos lingüísticos de mezcla de lenguas porque el léxico relacionado con el fútbol español está normalmente en francés, tenemos referencias constantes a los equipos españoles. En los Recortes 6 y 7, el Barça y sustantivos y adjetivos relacionados con el equipo catalán participan activamente del nombre que eligen aficionados africanos al fútbol. Seguidores de la página de Facebook del Coton Sport de Garoua, equipo del norte del país y de religión mayoritariamente musulmana, se identifican añadiendo a sus nombres árabes típicos de la zona un adjetivo o sustantivo ligado al Barcelona. La identificación del club barcelonés y Cataluña está patente en el corpus, a pesar de que esta identificación parece haberse diluido con el nuevo carácter global del club, redefinido como una empresa global de entretenimiento según recogen Ginesta et al. (2020).

Así, uno de los hinchas más fieles del CSG, considerado fan destacado del equipo por el administrador del grupo de aficionados en esta red social, ha añadido le Catalan en francés a su nombre en Facebook. Otro aficionado del mismo equipo añade Barcelona en el Recorte 7.

RECORTE 6. Seguidor del CSG

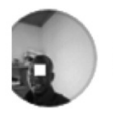

\section{A-Paul le Catalan}

RECORTE 7. Seguidor del CSG

\section{Sadam Barcelona}

En los ejemplos de esta última sección, se aprecia cómo las tecnologías de la información facilitan la formación de espacios virtuales que se extienden más allá de las fronteras tradicionales, culturales, lingüísticas o geográficas donde las relaciones entre los miembros de esa comunidad determinada o espacio virtual negocian su identidad, en muchos casos, construyendo una identidad idealizada con léxico que ensalza aún más esa falsa realidad individual de éxito. La construcción de esa identidad se crea adoptando léxico 
del fútbol global y adaptándolo a sus condiciones locales, es decir, los usuarios adoptan ideas del fútbol global que mezclan con sus nombres reales o su realidad local camerunesa, creando así una identidad glocal, o mezcla de ambas identidades, en línea con lo que indica Chiweshe (2014) sobre identidades locales y globales en las comunidades en Facebook de hinchas de fútbol de Zimbabue. Lo importante, en nuestro estudio, es que esa nueva identidad glocal está ligada, en muchos casos, al español.

\section{DISCUSIÓN Y CONCLUSIONES}

Antes de la llegada masiva de la red a nuestras vidas, Hernández (2003) comentaba que los neologismos estaban invadiendo el español, debido a lo fácil que les resultaba a estos términos incorporarse al lenguaje deportivo, al emplearlos las agencias internacionales y las publicaciones de tiradas millonarias. Este autor hablaba ya entonces de «... un lenguaje deportivo internacional cada día más conseguido y practicado»(2003: 87). Aquí, aún no se puede hablar de un lenguaje internacional, pero los resultados corroboran la abrumadora influencia que los equipos de fútbol españoles y el discurso que los acompaña tienen en lugares tan alejados de España como Camerún, como ejemplo claro de globalización lingüística.

No hay duda de que el lenguaje deportivo se caracteriza por su creatividad, especialmente en cuanto al léxico (Hernández 2012; Guerrero 2018), como vehículo de emociones que el periodista o el aficionado quieren transmitir, y tampoco hay duda de que varios géneros electrónicos adolecen de creatividad lingüística endémica, como recoge Chovanec (2021). Los resultados demuestran que la creatividad también va ligada a la mezcla de lenguas, práctica discursiva habitual en las conversaciones diarias de muchos países multilingües, también generalizada ahora en textos escritos de carácter público. En el corpus, los elogios y cumplidos típicos del país africano que tienen que ver con la familia se expresan en español para enfatizar el afecto hacia el ídolo, función frecuente del CC (por ejemplo, Androutsopoulos 2013).

Además, se aprecia la identificación de los internautas con las estrellas del fútbol español mediante la apropiación total o parcial de sus nombres, acompañados de calificativos laudativos o de cortesía positiva en estas lenguas extranjeras, lo que refuerza la admiración hacia el ídolo, su lengua y cultura, emblemas de la realización personal y social a la que estos aficionados parecen aspirar y que hoy en día representan los futbolistas (Hernández 2012). Asimismo, la apropiación de léxico relacionado con el club admirado subraya la identificación del internauta con su equipo campeón, modelo a seguir. 
Como se indicaba en la introducción, en referencia a Fairclough (2003), es patente la colonización o apropiación del lenguaje futbolístico español en la sociedad camerunesa o al menos, en el conjunto de aficionados al fútbol del país africano. El fútbol español ha conseguido convertirse en los últimos tiempos en un entretenimiento global con una audiencia global. Los clubes más conocidos de España se han centrado en conseguir este objetivo durante los últimos años (ver Ginesta et al. 2020) con campañas de promoción en otros continentes, pero, sobre todo, gracias a la tecnología, televisión vía satélite y redes sociales (Onwumechili y Akindes 2014). Así, la audiencia global ha posibilitado que las lenguas de estos equipos alcancen el carácter global que hemos visto en el corpus, lo que resulta novedoso y de interés para los estudios de la lengua española en la actualidad, aunque se trate solo de pequeñas combinaciones léxicas. No se puede corroborar la hipótesis de Pannenborg (2012) de que África está sufriendo una segunda colonización económica mediante el fútbol porque el tema de esta investigación no analiza los aspectos económicos del deporte global, pero sí se puede afirmar que existe cierta colonización o, más bien, apropiación discursiva en el continente, el cambio de código a estas lenguas da fe de esta tendencia. En otros campos, Hatalon (2015) certifica que la adopción de nombres en español para antropónimos es una estrategia relativamente frecuente en el Camerún actual, gracias a la universalización de la cultura hispánica por el fútbol y las telenovelas latinoamericanas.

En definitiva, los resultados de este estudio se engloban en dos puntos importantes. Por una parte, se observa la voluntad de estos internautas de identificarse con los éxitos del deporte español e italiano mediante el uso de léxico en las lenguas de estos países que parecen encarnar las cualidades que desean conseguir los aficionados cameruneses. Se da en esto cierto paralelismo con lo que ocurría en la antigua Roma con la inclusión de léxico en griego en textos predominantemente en latín: el cambio de código a esta lengua mostraba la apropiación por parte de los romanos de la lengua que consideraban de la alta cultura, como muestran las Cartas a Ático de Cicerón (Swain 2002). El español, italiano y catalán, pero sobre todo el español, parece configurarse actualmente como la lengua extranjera que representa la alta cultura en el fútbol, la lengua del éxito futbolístico al que aspiran los equipos cameruneses y sus seguidores.

Por otra parte, los resultados muestran que estos comentarios en Facebook conforman el espacio translingual del que hablaba Li (2011), donde los internautas usan todos los recursos lingüísticos disponibles para redactar sus comentarios, bien se trate de lenguas, sin importar el grado de conocimiento que el resto de participantes en cada comunidad de aficionados tengan de esa lengua o lenguas, como de los recursos típicos de la comunicación electrónica, por ejemplo, la reduplicación de signos de puntuación. 
Estos recursos enriquecen el texto escrito, dotándolo de las pistas paralingüísticas necesarias para suplir la ausencia del canal visual y que caracterizan al discurso electrónico (ver, por ejemplo, Georgakopoulou 1997; Yus 2011).

Sin lugar a dudas, el artículo ha demostrado que el cambio de código es una estrategia que marca la apropiación discursiva en el lenguaje público sobre fútbol por parte de internautas cameruneses. La mezcla de lenguas y la creatividad léxica que caracterizan el discurso oral de la sociedad camerunesa también es una característica relevante de sus conversaciones escritas sobre fútbol, donde el español tiene un papel destacado. Estudios futuros pueden analizar este fenómeno lingüístico en la conversación y en las áreas claves de la sociedad camerunesa identificadas por Anchimbe (2016), la música y la religión. El examen de textos privados intercambiados a través de mensajería instantánea, Facebook Messenger o WhatsApp, también podría aportar datos de interés académico sobre apropiación discursiva mediante CC a estas lenguas románicas, pero, esta vez, en entornos semiprivados de comunicación. 


\section{BIBLIOGRAFÍA}

Alba NiÑo, María (2015): «Switching, Mixing and Choosing Languages Online. The Case of Asturian and Castilian (Spanish)». En María Alba Niño y Rolf Kailuweit (eds.), Medien für Minderheitensprachen. Mediensprachliche Überlegungen zur Eintwicklung von Minderheitensprachen, Friburgo: Rombach Verlag KG, 211251.

Arias Alvárez, Alba (2017): «Cambio de código en la red: la expresión de la identidad en Asturias». En Mabel Giammatteo, Patricia Gubitosi y Alejandro Parini (eds.), El español en la red, Madrid: Vervuert Iberoamericana, 123-152.

Aleixandre-Benavent, Rafael, Recaredo Agulló Albuixech, Víctor Agulló CalaTAYUd y Juan Carlos VALDERRAMA-Zurián (2017): «Terminología y lenguaje deportivo del fútbol», Cultura, ciencia y deporte 2/6, 117-123.

Anchimbe, Eric A. (2013): Language Policy and Identity Construction: The Dynamics of Cameroon's Multilingualism, Ámsterdam y Filadelfia: John Benjamins.

- (2016): «Digital Narratives of Belonging as Anglophone or Francophone in a Cameroon Online News Forum», Open Linguistics 2/1, 511-527.

Androutsopoulos, Jannis (2013): "Code-switching in Computer-mediated Communication». En Susan C. Herring, Dieter Stein y Tuija Virtanen (eds.), Pragmatics of Computer-mediated Communication, Berlín y Boston: de Gruyter Mouton, 667-694.

- (2015): «Networked Multilingualism: Some Language Practices on Facebook and their Implications», International Journal of Bilingualism 19/2, 185-205.

ANTEZANA, Luis (2003): «Fútbol: espectáculo e identidad en América Latina». En Pablo Alabarces (ed.), Identidad, Futbol, Industria de la Cultura, Identidad Cultural, Deportes, Espectáculo, Buenos Aires: CLACSO, Consejo Latinoamericano de Ciencias Sociales, 85-97.

Atanga, Lilian L. (2012): «Language Choice, Identity, and Power in the Cameroonian Parliament». En Eric A. Anchimbe (ed.), The Linguistic and Social Context of English and Pidgin in Cameroon, Berlín: de Gruyter Mouton, 141-162.

Ayafor, Miriam y Melanie Green (2017): Cameroon Pidgin English: A Comprehensive Grammar (Vol. 20), Ámsterdam y Filadelfia: John Benjamins.

Baron, Naomi S. (2008): Always on: Language in an Online and Mobile World, Oxford: OUP.

- (2011): «Foreword». En Crispin Thurlow y Kristine Mroczek (eds.), Digital Discourse: Language in the New Media, Oxford: OUP, XI-XVI.

BRIz, Antonio (1990): «El proceso de sustantivación y lexicalización de los adjetivos con artículo en español», Filología Románica 7, 231-239.

Chrweshe, Manase K. (2014): «Online Football Fan Identities and Cyber-fandoms in Zimbabwe». En Chuka Onwumechili y Gerard Akindes (eds.), Identity and Nation in African Football, Londres: Palgrave Macmillan, 236-253. 
Chovanec, Jan (2021): “The Battle Fever's On”: Phrasal Routines and Multilingual Play in Sports Commentary». En Eva Lavric y Machteld Meulleman (eds.), Le corps et la langue du footballeur: match interdisciplinaire. Actes des journées d'études d'Innsbruck (2016) et de Reims (2018), Innsbruck: Innsbruck University Press.

Crolley, Liz (2008): «Using the Internet to Strengthen its Identity: The Case of Spanish Football», Sport in Society 11/6, 722-738.

FAIRCLOUgh, Norman (2003): Analysing Discourse: Textual Analysis for Social Research, Londres y Nueva York: Routledge.

- (2013): «Critical Discourse Analysis and Critical Policy Studies», Critical policy studies 7/2, 177-197.

Ferguson, Charles A. (1983): «Sports Announcer Talk: Syntactic Aspects of Register Variation», Language in society 12/2, 153-172.

García, Ofelia (2009): «Education, Multilingualism and Translanguaging in the 21st Century». En Tobe Skutnabb Kangas, Robert Phillipson, Ajit K. Mohanty y Minati Panda (eds.), Social Justice through Multilingual Education, Bristol, UK: Multilingual Matters, 140-158.

Georgakopoulou, Alexandra (1997): «Self presentation and Interactional Alliances in e mail Discourse: The Style- and Code switches of Greek Messages», International Journal of Applied Linguistics 7/2, 141-164.

GeORGalou, Mariza (2017): Discourse and Identity on Facebook: How We Use Language and Multimodality to Present Identity Online, Londres: Bloomsbury.

Ginesta, Xavier, Jordi de SAn Eugenio, Pau Bonet y Martí Ferrer (2020): «Global Football in the US Market. The Internationalization of FC Barcelona and its Media Coverage», Soccer \& Society 21/2, 209-224.

GonzÁlez RAmallal, Manuel E. (2014): «Prensa deportiva e identidad nacional: España en el Mundial de fútbol de Sudáfrica 2010», Política y Sociedad 51/2, 337-366.

Guerrero Salazar, Susana (2018): Creatividad y juego en el discurso deportivo de la prensa: aportaciones léxico-semánticas, Madrid: Arco/Libros.

GuMPERZ, John J. (1977): «The Sociolinguistic Significance of Conversational Code-switching», RELC Journal 8/2, 1-34.

Hatalong BoHo, Zacharie (2015): «Los nombres propios hispánicos en Camerún: entre aventura y globalización semiolingüística», Cuadernos de Lingüistica Hispánica 26, 89-102.

HeRnández Alonso, Néstor (2003): El lenguaje de las crónicas deportivas, Madrid: Cátedra.

- (2012): Tendencias en el lenguaje deportivo actual, Madrid: Visión Libros.

Herring, Susan C. (2007): «A Faceted Classification Scheme for Computermediated Discourse», Language@Internet 4/1.

HinRICHS, Lars (2016): «Modular Repertoires in English-using Social Networks: A Study of Language Choice in the Networks of Adult Facebook Users». En Lauren Squires (ed.), English in Computer-mediated Communication. Variation, Representation, and Change, Berlín: de Gruyter Mouton, 17-42.

JACKSON, Jamie (2014, diciembre 9): «Twitter, Facebook, Instagram: Who are the World's most Popular Football Clubs?», The Guardian. 
KүтӧLÄ, Samu (2014): «Negotiating Multilingual Discourse in a Finland-based Online Football Forum: Metapragmatic Reflexivity on Intelligibility, Expertise and 'Nativeness'». En Janus Møller y Lian Madsen (eds.), Globalisering, sprogning og normativitet: online og offline, The Copenhagen Studies in Bilingualism, Copenhague: Universidad de Copenhague, 81-122.

LeE, Carmen (2016): «Multilingual Resources and Practices in Digital Communication». En Alexandra Georgakopoulou y Tereza Spilioti (eds.), The Routledge Handbook of Language and Digital Communication, Londres y Nueva York: Routledge, 118-132.

- (2017): Multilingualism Online, Londres y Nueva York: Routledge.

Leppänen, Sirpa y Saija Peuronen (2012): «Multilingualism on the Internet». En Marilyn Martin-Jones, Adrian Blackledge y Angela Creese (eds.), The Routledge Handbook of Multilingualism, Londres y Nueva York: Routledge, 384-402.

LI, Wei (2011): «Moment Analysis and Translanguaging Space: Discursive Construction of Identities by Multilingual Chinese Youth in Britain», Journal of Pragmatics 43, 1222-1235.

Llopis-Goig, Ramón (2008): «National Orientation, Universal Outlook - The Symbolic Capital of FC Barcelona in the Global Era», European Journal for Sport and Society 5/1, 63-71.

MAR-Molinero, Clare (2006): «Forces of Globalisation in the Spanish-Speaking World: Linguistic Imperialism or Grassroots Adaptation». En Miranda Stewart y Clare Mar-Molinero (eds.), Globalization and Language in the Spanish-speaking World, Basingstoke: Palgrave Macmillan, 8-26.

MAYNOR, Natalie (1994): «The Language of Electronic Mail: Written Speech?». En Greta D. Little y Michael Montgomery (eds.), Centennial Usage Studies, Tuscaloosa: Alabama University Press, 48-54.

Montes-Alcalá, Cecilia (2016): «iSwitch: Spanish-English Mixing in Computermediated Communication», Journal of Language Contact 9/1, 23-48.

Mulo FARENKIA, Bernard (2011): «Formes de "mise à distance" de l'altérité ethnique au Cameroun», Journal of Pragmatics 43/6, 1484-1497.

Negrón Goldbarg, Rosalyn (2009): «Spanish-English Codeswitching in email Communication», Language@Internet 6/3.

Pannenborg, Arnold (2012): Big Men Playing Football: Money, Politics and Foul Play in the African Game, Tesis Doctoral, Leiden: African Studies Centre.

PAOliLlo, John C. (2007): «How Much Multilingualism? Language Diversity on the Internet». En Brenda Danet y Susan C. Herring (eds.), The Multilingual Internet: Language, Culture, and Communication Online, Nueva York: OUP, 408-430.

PAPACHARISSI, Zizi (2009): «The Virtual Geographies of Social Networks: A Comparative Analysis of Facebook, LinkedIn and A Small World», New Media \& Society 11/1-2, 199-220.

PÉnARd, Thierry, Nicolas Poussing, Blaise Mukoko y George B. T. PiAPtie (2015): «Internet Adoption and Usage Patterns in Africa: Evidence from Cameroon», Technology in Society 42, 71-80.

Pérez-Sabater, Carmen, Gemma Peña-Martínez, Ed Turney y Begoña MonteroFlETA (2008): «A Spoken Genre Gets Written: Online Football Commentaries in English, French, and Spanish», Written Communication 25/2, 235-261. 
- y Ginette Maguelouk-Moffo (2018): «El discurso público de la universidad: un estudio socio pragmático contrastivo de Camerún, España y Francia», Círculo de Lingüistica Aplicada a la Comunicación 73, 177-196.

— y - (2019): «Managing Identity in Football Communities on Facebook: Language Preference and Language Mixing Strategies», Lingua 225, 32-49.

— y - (2020): «Online Multilingualism in African Written Conversations», Studies in African Linguistics 49/1, 141-159.

Rojas TORRIJOS, José Luis (2012): «La futbolización de la información deportiva: un estudio de casos de cuatro diarios deportivos europeos», Comunicação \& Cultura 13, 77-95.

SIMPSON, Andrew (ed.) (2008): «Introduction», Language and National Identity in Africa, Oxford: OUP, 1-25.

SwaIn, Simon (2002): «Bilingualism in Cicero?». En James N. Adams, Mark Janse y Simon Swain (eds.), Bilingualism in Ancient Society: Language Contact and the Written Text, Oxford: OUP, 128-167.

VidACS, Bea (1999): «Football in Cameroon: A Vehicle for the Expansion and Contraction of Identity», Culture, Sport, Society 2/3, 100-117.

Warschauer, Mark e Inez De Florio-Hansen (2003): «Multilingualism, Identity, and the Internet». En Adelheid Hu e Inez De Florio-Hansen (eds.), Multiple Identity and Multilingualism, Tubinga: Stauffenburg, 155-179.

Yus, Francisco (2011): Cyberpragmatics: Internet-mediated Communication in Context, Ámsterdam y Filadelfia: John Benjamins. 